\section{Multiple stenting in a patient with a high-grade malignant hilar biliary stricture: endoscopic four-branched partial stent-in-stent deployment of metallic stents}

Palliative therapy, such as biliary stenting, is often required before chemotherapy in patients with cholangiocellular carcinoma, who often have a hilar biliary stricture. In addition, chemotherapy itself puts patients at a higher risk of cholangitis. Drainage of both hepatic lobes is more advantageous than drainage of a single lobe alone, to avoid the development of cholangitis in the other lobe [1,2], but the deployment of multiple stents in a hilar biliary stricture is technically demanding. We report here the endoscopic four-branched partial stent-in-stent deployment of metallic stents in a patient with a Bismuth type IV hilar biliary stricture.

A 64-year-old man was admitted to our hospital with a liver tumor, dilatation of the intrahepatic bile ducts, and abnormal liver function tests. He had no jaundice at the time of admission, but his serum alkaline phosphatase was raised at 947 IU/ L. Endoscopic cholangiography showed that he had a Bismuth type IV stricture ( $\bullet$ Fig. 1). Furthermore, in the anterior segment of the liver the bile ducts were stenotic at the bifurcation of S5 and S8 due to tumor invasion. Because the patient had para-aortic lymph node metastasis, we judged that the tumor was unresectable and that he should receive chemotherapy. Before chemotherapy, we placed four JoStent SelfX stents (Abbott Vascular Devices, Redwood City, California, USA) - in the left lateral segment, in S5 and S8 of the right anterior segment, and in the right posterior segment - using the partial stent-in-stent procedure ( $\bullet$ Fig. 2). To facilitate placement, we first identified the target branches and inserted 0.025 -inch guide wires into them as landmarks for the bifurcation ( $\bullet$ Fig. 3). The patient was discharged and began chemotherapy with gemcitabine as an outpatient. He lived for more than 1 year without jaundice. This case confirms that the JoStent SelfX is suitable for the management of high-grade malignant hilar biliary stricture [3].
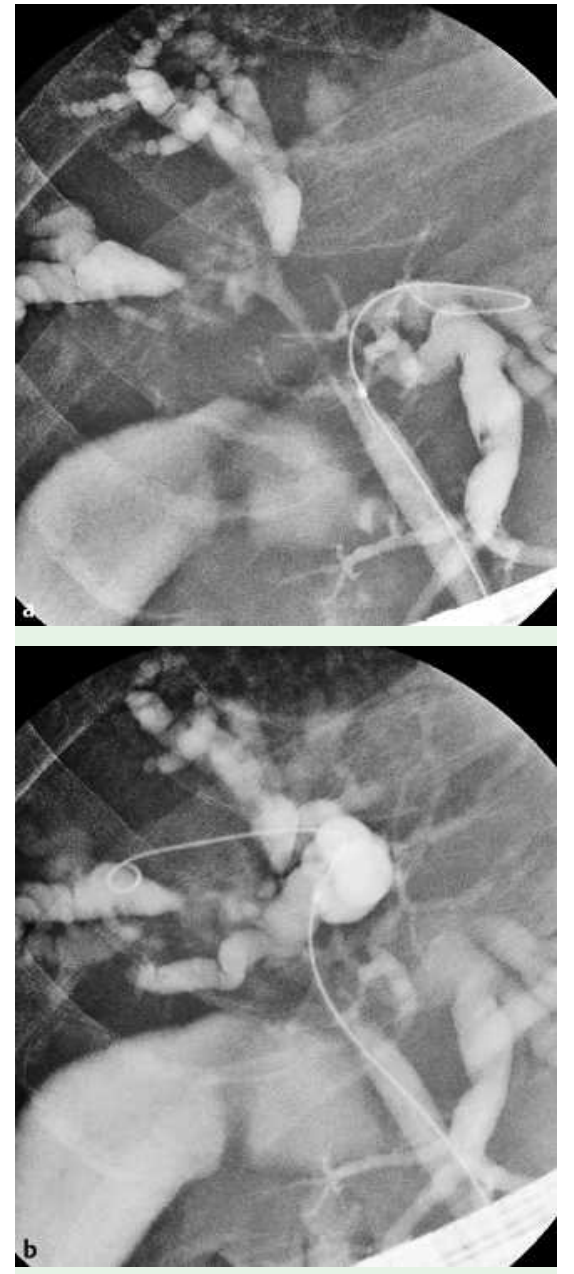

Fig. 1 a Cholangiography showed the left hepatic duct and the right anterior branch of the right hepatic duct. The bifurcation of the right anterior branch at S5 and S8 was invaded by the tumor. The right posterior branch is not opacified in this figure. b After identifying the right posterior branch with the guide wire, the cannula was advanced and the branch was visualized.

H. Kawamoto, K. Tsutsumi, M. Fujii, R. Harada, H. Kato, K. Hirao, N. Kurihara, T. Nakanishi, O. Mizuno, E. Ishida, T. Ogawa, H. Fukatsu, K. Sakaguchi Department of Gastroenterology and Hepatology, Okayama University Graduate School of Medicine and Dentistry, Okayama City, Japan

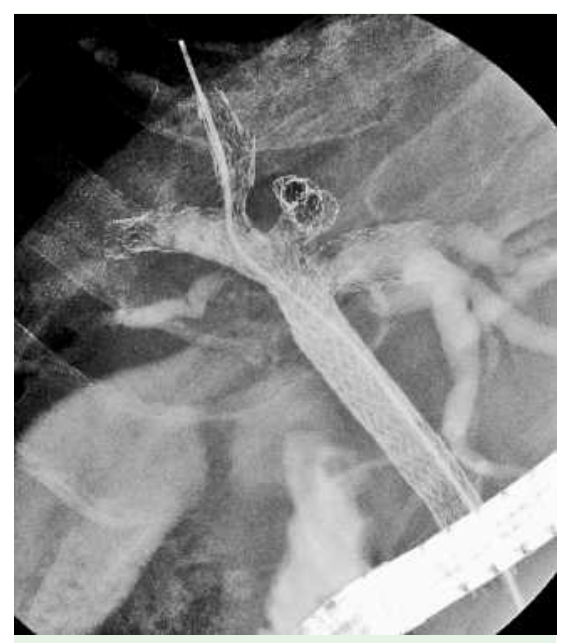

Fig. 2 Four JoStent SelfX stents were deployed - in the left lateral segment, the right posterior segment, and in the S5 and S8 branches of the right anterior segment branch, using the partial stent-in-stent procedure.
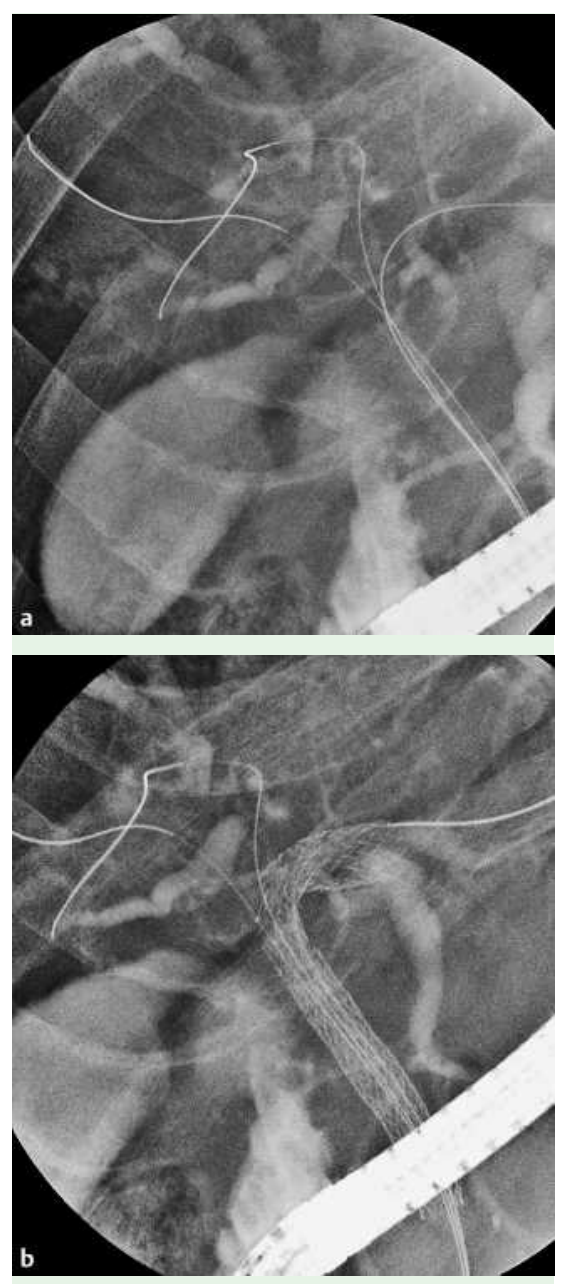

Fig. 3 a Three landmark guide wires were inserted in the left lateral, right anterior, and right posterior segments. $\mathbf{b}$ The first JoStent SelfX was deployed in the left hepatic duct. Two guide wires became landmarks of the bifurcation for identifying the anterior branch or the posterior branch of the right hepatic duct through the stent mesh. 


\section{References}

1 Devière J, Motte S, Dumonceau JM et al. Septicemia after endoscopic retrograde cholangiopancreatography. Endoscopy 1990; 22: $72-75$

2 Dumas R, Demuth N, Buckley M et al. Endoscopic bilateral metal stent placement for malignant hilar stenosis: identification of optimal technique. Gastrointest Endosc 2000; 51: 334-338

3 Kawamoto H, Ishida E, Ogawa T et al. Multiple stenting in hilar bile duct carcinoma: three-branched partial stent-in-stent deployment with the JOSTENT SelfX. Endoscopy $2004 ; 36: 829-830$

\section{Bibliography}

DOI $10.1055 / \mathrm{s}-2007-966612$

Endoscopy 2007; 39: E167-E168

(c) Georg Thieme Verlag KG Stuttgart · New York . ISSN 0013-726X

\section{Corresponding author}

\section{H. Kawamoto, MD}

Department of Gastroenterology and Hepatology Okayama University Graduate School of Medicine and Dentistry

2-5-1 Shikata-cho

Okayama City 700-8558

Japan

Fax: +81-86-225-5991

h-kawamo@md.okayama-u.ac.jp 